Acta Crystallographica Section B

Structural

Science

ISSN 0108-7681

Pamela A. McGregor, ${ }^{\mathrm{a}, \mathrm{b}}$ David R. Allan, ${ }^{b, c *}$ Simon Parsons ${ }^{b, c}$ and Colin R. Pulham ${ }^{\mathrm{b}, \mathrm{c}}$

a'School of Physics and Astronomy, The Univer-
sity of Edinburgh, King's Buildings, West Mains
Road, Edinburgh EH9 3JZ, Scotland, 'Centre for
Science at Extreme Conditions, The University of
Edinburgh, King's Buildings, West Mains Road,
Edinburgh EH9 3JZ, Scotland, and 'School of
Chemistry, The University of Edinburgh, King's
Buildings, West Mains Road, Edinburgh EH9 3JJ,
Scotland

Correspondence e-mail: d.r.allan@ed.ac.uk

\section{The low-temperature and high-pressure crystal structures of cyclobutanol $\left(\mathrm{C}_{4} \mathrm{H}_{7} \mathrm{OH}\right)$}

The low-temperature and high-pressure crystal structures of cyclobutanol $\left(\mathrm{C}_{4} \mathrm{H}_{7} \mathrm{OH}\right)$ have been determined using singlecrystal X-ray diffraction techniques. At temperatures below $220 \mathrm{~K}$, cyclobutanol crystallizes in the $A b a 2$ space group $\left(Z^{\prime}=\right.$ 2 ) and its crystal structure is composed of pseudo-threefold hydrogen-bonded molecular catemers [assigned as $C_{2}^{2}(4)$ in graph-set notation], which lie parallel to the crystallographic $a$ axis. At a pressure of $1.3 \mathrm{GPa}$, the crystal symmetry changes to $\operatorname{Pna2}_{1}\left(Z^{\prime}=1\right)$ and the molecular catemers [expressed as $C(2)$ in graph-set notation] adopt a pseudo-twofold arrangement. This structural behaviour is in agreement with our previous observations for phenol and its halogenated derivatives 2chlorophenol and 4-fluorophenol, where pressure was found to favour a molecular packing more closely associated with small alkyl groups rather than that of relatively bulky alkyl groups. In addition, an examination of the molecular coordination environment in the low-temperature and highpressure structures of cyclobutanol reveals that the change in structure on application of pressure appears to be driven by the molecules assuming a packing arrangement which more closely resembles that adopted in hard-sphere structures.

\section{Introduction}

In the mono-alcohols $(R \mathrm{OH})$ there is competition between the packing requirements of the relatively bulky $R$ group and the demand for the small hydroxyl groups to be sufficiently close for hydrogen bonding to occur. Brock \& Duncan (1994) have described the general features of the packing motifs adopted by mono-alcohols. They found that if the molecules containing the hydroxyl groups are relatively 'thin' (by Brock and Duncan's terminology) then they can form catemers where the molecules are symmetry-related by either a glide plane or a $2_{1^{-}}$ screw axis so that the molecules form an approximately coplanar alternating sequence about the central chain of hydrogen bonds. For bulkier $R$ groups, steric hindrance often prohibits the molecules adopting this simple arrangement and, instead, these systems often form chains about three-, four- or sixfold screw axes, or adopt crystal structures with more than one molecule in the asymmetric unit. If the $R$ group is particularly bulky, then the molecules may no longer form hydrogen-bonded chains or catemers, but cyclic dimer, trimer, tetramer or hexamer rings can be created.

In our recent high-pressure structural studies of phenol (Allan et al., 2002) and its halogenated derivatives 2-chlorophenol and 4-fluorophenol (Oswald et al., 2005), we have observed a clear change in the nature of the $R$-group packing behaviour. All three systems form crystal structures at ambient pressure, characterized by the formation of hydrogen-bonding schemes associated with bulky $R$ groups.
Received 19 January 2005

Accepted 16 June 2005
(C) 2005 International Union of Crystallography Printed in Great Britain - all rights reserved 
Both phenol and 2-chlorophenol form crystal structures where the molecules are hydrogen bonded into pseudo-threefold chains. The ambient-pressure structure of 4-fluorophenol has a markedly different packing arrangement with the molecules hydrogen bonding to form hexamer rings about threefold rotoinversion sites. After the application of pressure, however, all three systems form crystal structures with the molecules disposed along chains that are generated by $2_{1}$ screw axes. In effect, pressure has transformed the packing behaviour of the phenyl and halophenyl groups from having characteristics more closely associated with bulky groups to those more typical of small groups.

Here we report the crystal structure of the high-pressure phase of cyclobutanol $\left(\mathrm{C}_{4} \mathrm{H}_{7} \mathrm{OH}\right)$ along with the crystal structure of its low-temperature phase, which, to the best of our knowledge, has not been reported previously. The lowtemperature crystal structure (space group $A b a 2, Z^{\prime}=2$ ) is composed of pseudo-threefold hydrogen-bonded molecular catemers which lie parallel to the crystallographic $a$-axis. At high-pressure, the crystal symmetry changes to $\operatorname{Pna}_{1}\left(Z^{\prime}=1\right)$ and the molecular catemers, which are generated by the $a$ glide symmetry, adopt a pseudo-twofold arrangement. This structural behaviour parallels what we have observed previously for phenol and 2-chlorophenol and is in agreement with the favouring of a small-group packing behaviour under pressure.

\section{Experimental}

Cyclobutanol $\left(\mathrm{C}_{4} \mathrm{H}_{8} \mathrm{O}\right)$ is a clear, colourless liquid with a melting point of $221 \mathrm{~K}$ and a boiling point of $395 \mathrm{~K}$. It is a secondary alcohol which consists of four $s p^{3}$ hybridized $\mathrm{C}$ atoms arranged in a puckered four-membered ring. The puckering of the ring increases the angle strain in the molecule, but relieves the eclipsing interactions of adjacent $\mathrm{C}-\mathrm{H}$ bonds.

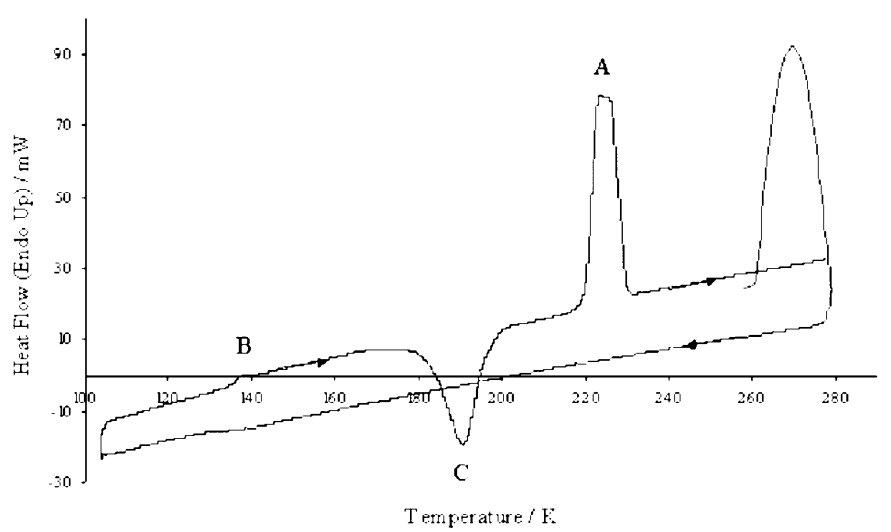

Figure 1

The relationship between heat flow versus temperature for cyclobutanol. The sample was loaded into the differential scanning calorimeter as a liquid at room temperature, $273 \mathrm{~K}$. The sample temperature was cycled from 273 to $105 \mathrm{~K}$ and finally back to room temperature at a rate of $10 \mathrm{~K} \mathrm{~min}^{-1}$. $A=$ melting, $B=$ glass transition, $C=$ crystallization of glass.

\subsection{Differential scanning calorimetry (DSC)}

Before proceeding with structure characterization using single-crystal X-ray diffraction methods, a survey of the lowtemperature phase behaviour of cyclobutanol was undertaken using a Perkin-Elmer Pyris differential scanning calorimeter DSC-1. The sample of cyclobutanol $(99.5 \%$, obtained from Sigma-Aldrich) was contained in a sealed aluminium pan. Fig. 1 shows that super-cooling occurs in the sample and the liquid does not crystallize until it is warmed where the exothermic crystallization peak occurs at $180 \mathrm{~K}$ (event $C$ in Fig. 1). The small endothermic event which occurs in this first scan at approximately $138 \mathrm{~K}$ (event $B$ in Fig. 1) is a glass transition in which some degree of ordering occurs in the glassy phase. This does not occur in the polycrystalline material. Melting occurs at $221 \mathrm{~K}$ (event $A$ in Fig. 1). The DSC experiment showed that no phase change occurs in the material down to temperatures as low as $105 \mathrm{~K}$ after initial crystallization. Therefore, a single crystal could be grown at a temperature just below the $221 \mathrm{~K}$ melting point and then safely cooled for subsequent X-ray data collection.

\subsection{Low-temperature crystal growth of phase I}

Liquid cyclobutanol was loaded into a capillary of $0.33 \mathrm{~mm}$ diameter. This was attached to a goniometer head and mounted on a BRUKER SMART-APEX (Siemens, 1993) diffractometer, equipped with an Oxford Cryosystems lowtemperature device and an OHCD laser-assisted crystallization device (Boese \& Nussbaumer, 1994). The sample was cooled to just below the melting point. A solid-liquid equilibrium was established at approximately $1.5 \mathrm{~W}$ laser power and a crystal was grown by applying the same laser power along approximately $0.5 \mathrm{~mm}$ of the capillary over a duration of $30 \mathrm{~min}$. The laser power was subsequently reduced to $0 \mathrm{~W}$ over a further period of $20 \mathrm{~min}$ at the end of the cycle.

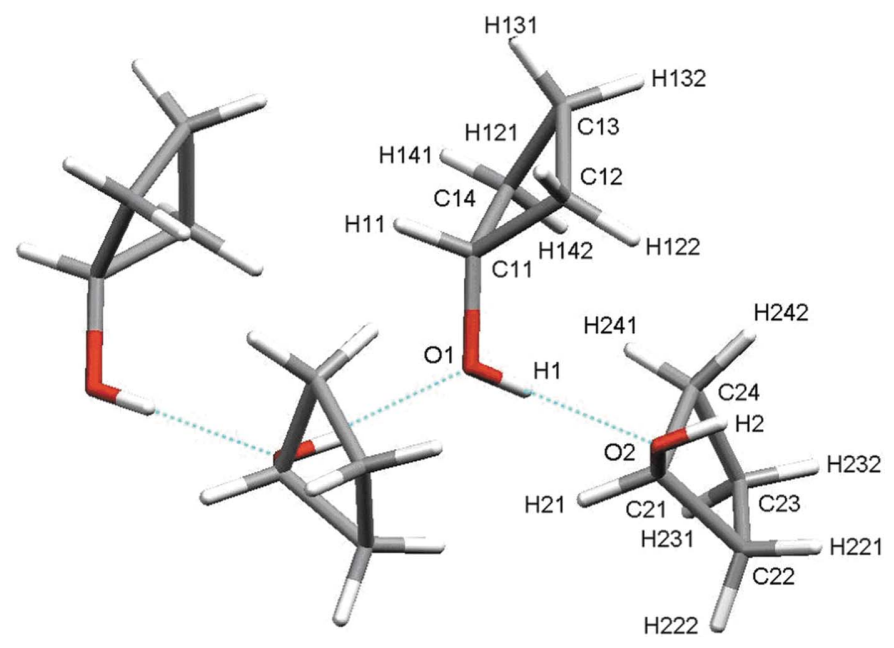

Figure 2

A view of the hydrogen-bonded molecular chains in the low-temperature, $A b a 2$, crystal structure of cyclobutanol. The chains are aligned parallel to the crystallographic $a$ axis. 
Table 1

Refinement statistics for the low-temperature, $A b a 2$, and high-pressure, $P n a 2_{1}$, phases of cyclobutanol.

\begin{tabular}{|c|c|c|c|}
\hline Temperature $(\mathrm{K})$; pressure $(\mathrm{GPa})$ & $220 ; 0$ & $100 ; 0$ & $293 ; 1.3$ \\
\hline \multicolumn{4}{|l|}{ Crystal data } \\
\hline Chemical formula & $\mathrm{C}_{4} \mathrm{H}_{8} \mathrm{O}$ & $\mathrm{C}_{4} \mathrm{H}_{8} \mathrm{O}$ & $\mathrm{C}_{4} \mathrm{H}_{8} \mathrm{O}$ \\
\hline$M_{r}$ & 72.10 & 72.10 & 72.10 \\
\hline Cell setting, space group & Orthorhombic, $A b a 2$ & Orthorhombic, $A b a 2$ & Orthorhombic, Pna2 1 \\
\hline$a, b, c(\AA)$ & $9.379(2), 13.658(2), 13.661(2)$ & $9.331(2), 13.642(2), 13.619(2)$ & $4.9208(4), 8.230(1), 9.598(2)$ \\
\hline$V\left(\AA^{3}\right)$ & $1749.9(5)$ & $1733.7(5)$ & $388.71(9)$ \\
\hline$Z$ & 16 & 16 & 4 \\
\hline$D_{x}\left(\mathrm{Mg} \mathrm{m}^{-3}\right)$ & 1.095 & 1.105 & 1.232 \\
\hline Radiation type & Mo $K \alpha$ & Mo $K \alpha$ & Mo $K \alpha$ \\
\hline No. reflections for cell parameters & 2278 & 3363 & 479 \\
\hline$\theta$ range $\left({ }^{\circ}\right)$ & $3-26$ & $3.8-28.4$ & $3-20$ \\
\hline$\mu\left(\mathrm{mm}^{-1}\right)$ & 0.08 & 0.08 & 0.09 \\
\hline Temperature (K) & $220(2)$ & $100(2)$ & $293(2)$ \\
\hline Crystal form, colour & Cylinder, colourless & Cylinder, colourless & Prism, colourless \\
\hline Crystal size $(\mathrm{mm})$ & $0.50 \times 0.33 \times 0.33$ & $0.50 \times 0.33 \times 0.33$ & $0.02 \times 0.02 \times 0.01$ \\
\hline \multicolumn{4}{|l|}{ Data collection } \\
\hline Diffractometer & CCD area detector & CCD area detector & CCD area detector \\
\hline Data collection method & $\varphi$ and $\omega$ scans & $\varphi$ and $\omega$ scans & $\varphi$ and $\omega$ scans \\
\hline Absorption correction & $\begin{array}{l}\text { Multi-scan (based on symmetry- } \\
\text { related measurements) }\end{array}$ & $\begin{array}{l}\text { Multi-scan (based on symmetry- } \\
\text { related measurements) }\end{array}$ & $\begin{array}{l}\text { Multi-scan (based on symmetry- } \\
\text { related measurements) }\end{array}$ \\
\hline$T_{\max }$ & 0.693 & 0.766 & 0.425 \\
\hline$T_{\min }$ & 1.000 & 1.000 & 0.928 \\
\hline $\begin{array}{l}\text { No. of measured, independent and } \\
\text { observed reflections }\end{array}$ & $4279,918,824$ & $5003,1093,922$ & $747,225,197$ \\
\hline Criterion for observed reflections & $I>2 \sigma(I)$ & $I>2 \sigma(I)$ & $I>2 \sigma(I)$ \\
\hline$R_{\text {int }}$ & 0.046 & 0.051 & 0.073 \\
\hline$\theta_{\max }\left({ }^{\circ}\right)$ & 26.4 & 28.6 & 20.0 \\
\hline Range of $h, k, l$ & $-11 \Rightarrow h \Rightarrow 11$ & $-12 \Rightarrow h \Rightarrow 12$ & $-4 \Rightarrow h \Rightarrow 4$ \\
\hline & $-17 \Rightarrow k \Rightarrow 14$ & $-17 \Rightarrow k \Rightarrow 14$ & $-7 \Rightarrow k \Rightarrow 7$ \\
\hline & $-14 \Rightarrow l \Rightarrow 17$ & $-17 \Rightarrow l \Rightarrow 15$ & $-6 \Rightarrow l \Rightarrow 6$ \\
\hline \multicolumn{4}{|l|}{ Refinement } \\
\hline Refinement on & $F^{2}$ & $F^{2}$ & $F^{2}$ \\
\hline$R\left[F^{2}>2 \sigma\left(F^{2}\right)\right], w R\left(F^{2}\right), S$ & $0.042,0.104,1.07$ & $0.047,0.106,1.00$ & $0.071,0.162,1.14$ \\
\hline No. of reflections & 918 & 1093 & 225 \\
\hline No. of parameters & 108 & 108 & 23 \\
\hline $\mathrm{H}$-atom treatment & Riding & Riding & Riding \\
\hline Weighting scheme & $\begin{array}{l}w=1 /\left[\sigma^{2}\left(F_{o}^{2}\right)+(0.0702 P)^{2}\right], \text { where } \\
\quad P=\left(F_{o}^{2}+2 F_{c}^{2}\right) / 3\end{array}$ & $\begin{array}{l}w=1 /\left[\sigma^{2}\left(F_{o}^{2}\right)+(0.0649 P)^{2}\right], \text { where } \\
\quad P=\left(F_{o}^{2}+2 F_{c}^{2}\right) / 3\end{array}$ & $\begin{array}{l}w=1 /\left[\sigma^{2}\left(F_{o}^{2}\right)+(0.0788 P)^{2}+\right. \\
\quad 0.4005 P], \text { where } P=\left(F_{o}^{2}+2 F_{c}^{2}\right) / 3\end{array}$ \\
\hline$(\Delta / \sigma)_{\max }$ & 0.051 & $<0.0001$ & $<0.0001$ \\
\hline$\Delta \rho_{\max }, \Delta \rho_{\min }\left(\mathrm{e} \AA^{3}\right)$ & $0.17,-0.17$ & $0.25,-0.22$ & $0.20,-0.19$ \\
\hline Extinction method & SHELXTL & SHELXTL & None \\
\hline Extinction coefficient & $0.013(2)$ & $0.0080(4)$ & - \\
\hline Absolute structure & Flack (1983) & Flack (1983) & Flack (1983) \\
\hline Flack parameter & $-10(10)$ & $-10(10)$ & $1(10)$ \\
\hline
\end{tabular}

Computer programs used: SMART (Siemens, 1993), SAINT (Siemens, 1995), SHELXTL, SHELXS97, SHELXL97 (Sheldrick, 2001).

A hemisphere of data was collected in the range $2 \theta<52^{\circ}$ and the resulting diffraction pattern was indexed using GEMINI (Sparks, 1999) and integrated with SAINT (Siemens, 1995). An absorption correction was applied using $S A D A B S$ (Sheldrick, 2001). The structure was initially solved by direct methods in the space group $C 2$ and the symmetry was later increased to $A b a 2$ after analysis with the program MISSYM, as incorporated into PLATON (Spek, 2001). The structure was refined by full-matrix least-squares against $\left|F^{2}\right|$ (SHELXTL; Sheldrick, 2001). Initially, the $\mathrm{H}$ atoms were located using difference Fourier maps and, in subsequent cycles of refinement, their positions were idealized and constrained geometrically. All non-H atoms were modeled with anisotropic displacement parameters and, as the data were of sufficient quality, the $\mathrm{H}$ atoms could be refined isotropically. The sample was then cooled to $100 \mathrm{~K}$ and a second set of intensity data were collected, following the same strategy as that employed for the $220 \mathrm{~K}$ data set. Refinement details and statistics are shown in Table $1 .^{\mathbf{1}}$

\subsection{High-pressure crystal growth of phase II}

Liquid cyclobutanol was loaded and pressurized in a Merrill-Bassett diamond-anvil cell (Merrill \& Bassett, 1974) equipped with $600 \mu \mathrm{m}$ culet diamonds and a tungsten gasket. After the nucleation of several crystallites the temperature was cycled close to the melting curve, in order to reduce the

\footnotetext{
${ }^{1}$ Supplementary data for this paper are available from the IUCr electronic archives (Reference: WS5024). Services for accessing these data are described at the back of the journal.
} 
Table 2

The donor-acceptor $\mathrm{O} \cdots \mathrm{O}$ distances in the low-temperature, $A b a 2$, and high-pressure, Pna $2_{1}$, crystal structures of cyclobutanol.

\begin{tabular}{lll}
\hline Phase I & $\mathrm{O} 2 \cdots \mathrm{O} 1(\AA)$ & $\mathrm{O} \cdots \mathrm{O} 2^{\mathrm{i}}(\AA)$ \\
\hline $220 \mathrm{~K}$ & $2.709(3)$ & $2.742(2)$ \\
$110 \mathrm{~K}$ & $2.703(3)$ & $2.736(2)$ \\
& $\mathrm{O} 1 \cdots \mathrm{O} 1^{\mathrm{i}}(\AA)$ & \\
Phase II & $2.809(4)$ & \\
$1.3 \mathrm{GPa}$ &
\end{tabular}

Symmetry code: (i) $1 x-\frac{1}{2},-y-\frac{1}{2}, z$.

number of crystallites. Finally, a single crystal was obtained at approximately $1.3 \mathrm{GPa}$ that entirely filled the $175 \mu \mathrm{m}$ gasket hole.

Data were collected with the cell mounted in a single orientation and the subsequent diffraction pattern was indexed with the program GEMINI (Sparks, 1999). Data integration (to $2 \theta=45^{\circ}$ ) was performed using $S A I N T$ (Siemens, 1995) with dynamic masking to account for the shading from the steel body of the diamond-anvil cell. The program SHADE (Parsons, 2004) was used to take account of absorption effects due to the pressure cell and further systematic errors were treated using $S A D A B S$ (Sheldrick, 2001) before merging in SORTAV (Blessing, 1997). More detailed data collection and processing procedures used in our laboratory have been described in Dawson et al. (2004). Structure solution and refinement procedures were similar to those outlined for the low-temperature data sets, with the exception that the $\mathrm{H}$-atom positions could not be observed in the Fourier difference maps and they had to be located and

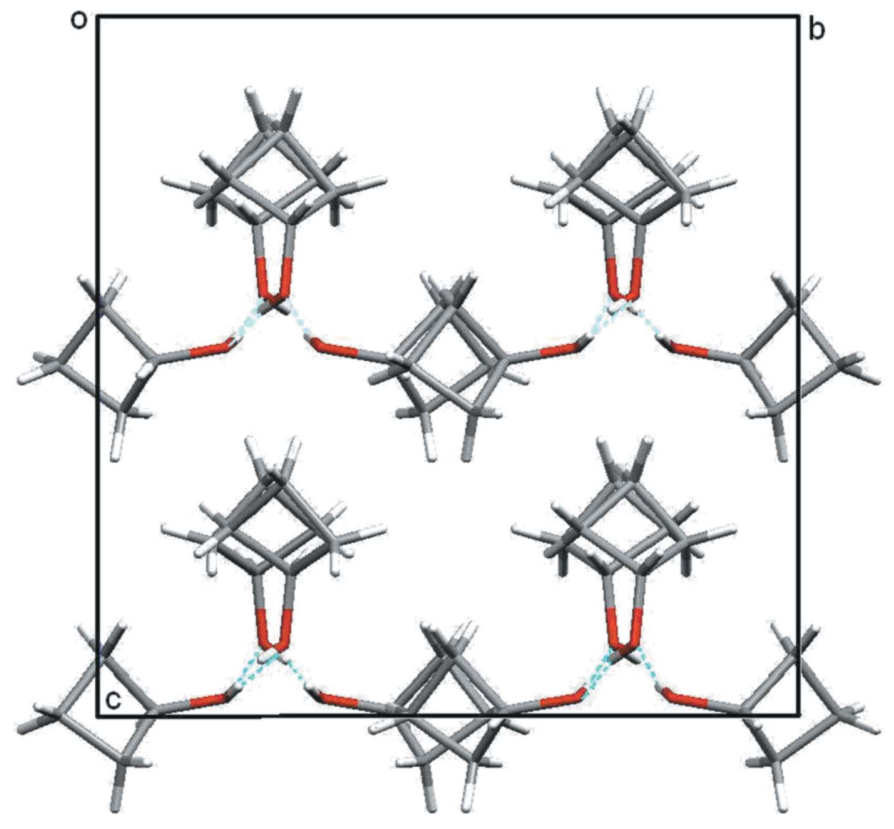

Figure 3

Packing plot of cyclobutanol phase I viewed parallel to the crystallographic $a$ axis showing the intertwining of the chains via neighbouring alkyl groups is readily apparent (the $b$ axis is directed towards the right of the page, the $c$ axis is directed down the page). constrained in the refinement using geometrical considerations. The final refinement statistics are listed in Table 1.

\section{Results}

\subsection{The low-temperature phase I crystal structure}

The crystal structure of phase I of cyclobutanol has two molecules in the asymmetric unit and it is characterized by the presence of binary hydrogen-bonded chains of cyclobutanol molecules aligned parallel to the crystallographic $a$ axis. The two molecules in the asymmetric unit are hydrogen bonded together to form a single section of the chain and the $b$-glide symmetry links these sections in pairs to construct the complete catemer. These catemers are not strictly helical in nature, as helices are not supported by the $b$ glide. The chains are assigned the graph-set notation $C_{2}^{2}(4)$ - having two unique hydrogen-bond donors and two unique hydrogen-bond acceptors involving a total of four atoms. Fig. 2 shows an individual hydrogen-bonded chain, while the complete structure is shown in projection along the $a$ axis in Fig. 3. From this $a$-axis projection, the pseudo-threefold nature of the chains is apparent and it can also be observed that the alkyl groups of molecules in neighbouring chains lie on top of one another to form an intertwining arrangement. The chains themselves are stacked in layers perpendicular to the crystallographic $b$ axis and, in the crystal structure as a whole, each molecule occupies a volume of 109.4 (1) $\AA^{3}$ at $220 \mathrm{~K}$ and 108.4 (1) $\AA^{3}$ at $100 \mathrm{~K}$ (a difference of $0.9 \%$ ).

\subsection{The high-pressure phase II crystal structure}

The crystal structure of phase II of cyclobutanol has one molecule in the asymmetric unit that acts dually as a hydrogen-bond donor and a hydrogen-bond acceptor. Consequently, there is one unique hydrogen bond in the structure and this links neighbouring molecules to form

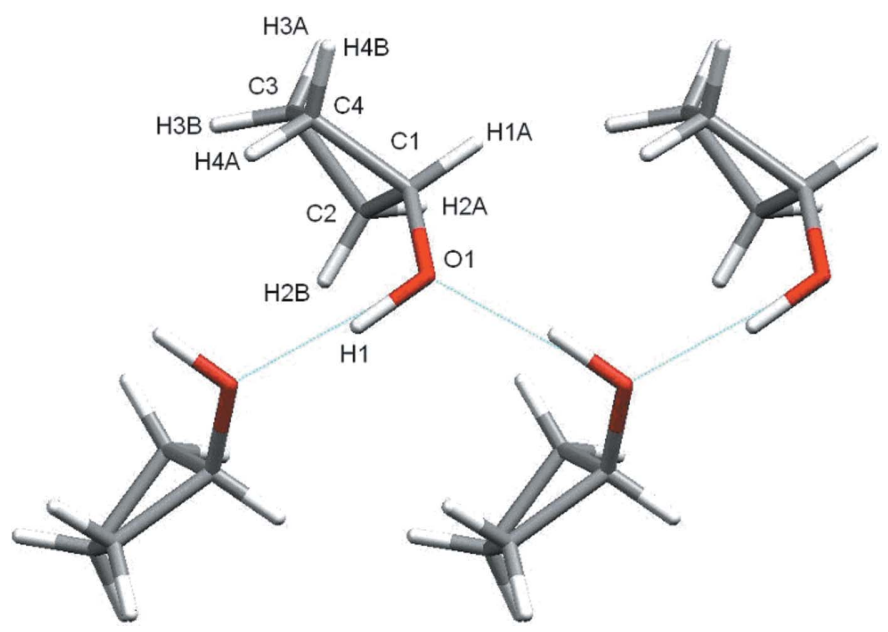

Figure 4

The wave-like hydrogen-bonded chains of the high-pressure, $P n a 2_{1}$, phase of cyclobutanol. The chains are aligned parallel to the crystallographic $a$ axis. 
hydrogen-bonded chains expressed as $C(2)$ in graph-set notation - the repeating unit contains one hydrogen-bond donor and one hydrogen-bond acceptor. At a pressure of 1.3 GPa, each molecule occupies $97.2(1) \AA^{3}$ and comparing this to the volume of the low-temperature phase gives a $10.3 \%$ reduction in volume with respect to the $100 \mathrm{~K}$ structure and an $11.2 \%$ reduction in volume compared with the $220 \mathrm{~K}$ structure.

Fig. 4 shows the hydrogen-bonded chains of cyclobutanol molecules. The chains involve a repeating unit of two molecules related by the $a$ glide symmetry. The wave-like chains are aligned in layers stacked along the $c$ axis, as shown in Fig. 5 and, unlike the chains in the low-temperature phase, the packing does not result in an intertwining of the alkyl groups.

\section{Discussion and comparison of the low-temperature and high-pressure phases}

Perhaps the most significant difference between the lowtemperature and the high-pressure polymorphs is the reduction in molecular volume. At ambient pressure and $220 \mathrm{~K}$, each molecule occupies $108.4(1) \AA^{3}$ compared with 96.8 (1) $\AA^{3}$ at room temperature and $1.3 \mathrm{GPa}$, a decrease of approximately $10 \%$. It would naturally be assumed that this reduction in molecular volume with pressure would be accommodated by the intermolecular contacts and that the hydrogen bonds should exhibit a strong pressure effect. This does not appear to be the case and the donor-acceptor distances for the low-temperature phase are somewhat shorter than those in the high-pressure phase, see Table 2. Although this observation is perhaps counter-intuitive, a similar affect also been observed on comparison of the crystal structures of the low-temperature and high-pressure phases of phenol (Allan et al., 2002) and of the halophenols (Oswald et al., 2005). It was suggested that the reason for the increase in the hydrogen-bond distance with pressure for the phenols was

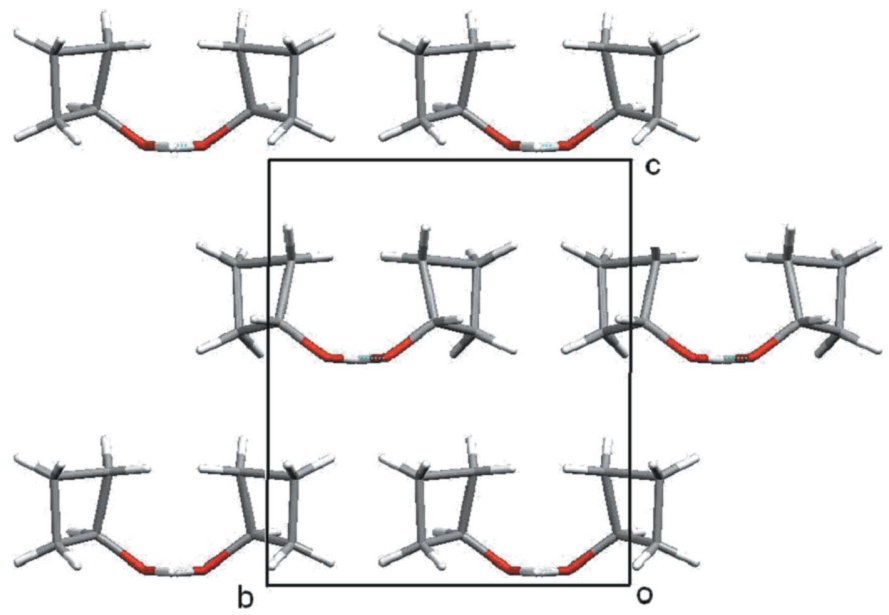

Figure 5

Packing plot of the high-pressure phase of cyclobutanol viewed down the crystallographic $a$ axis (the $b$ axis is directed towards the left of the page, the $c$ axis is directed up the page). due, principally, to steric effects. The observation that there is a similar effect in cyclobutanol provides further evidence that this lengthening of the hydrogen bond is linked to steric considerations.

It is interesting to note that the apparent increase in hydrogen-bond length between the high-pressure and lowtemperature phases of cyclobutanol, phenol and the halophenols is paralleled by strikingly similar changes to the arrangement of the molecules themselves within the hydrogen-bonded chains. The a-glide which generates the hydrogen-bonded molecular chains in both the low-temperature, $A b a 2$, and high-pressure, Pna2 ${ }_{1}$, crystal structures, is basically a twofold symmetry operation. Brock \& Duncan (1994) have demonstrated that small molecules pack along $2_{1}$ screw axes or glides, and hence the high-pressure behaviour of cyclobutanol is typical of them. An analogous structural transformation is also observed in phenol, where the same topological change from pseudo-threefold to pseudo-twofold occurs between the low-temperature and high-pressure phases, respectively (where both polymorphs have the same $P 2_{1}$ space group). This trend is also observed in three halophenol systems studied by Oswald et al. (2005). Although they form crystal structures at ambient pressure which are typical of molecules containing bulky $R$ groups, at high pressure they adopt crystal structures with the molecules disposed along chains that are generated by $2_{1}$ screw axes.

Finally, given that the reduction in molecular volume at pressure cannot be attributed to the shortening of intermolecular hydrogen bonding, and indeed the converse appears to be the case, it would be valuable, therefore, to compare other structural features, such as the molecular

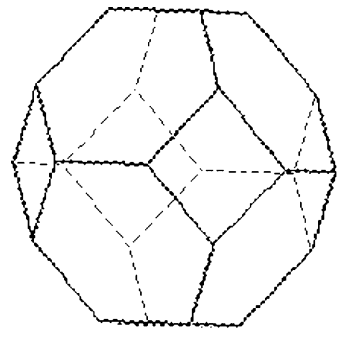

(a)

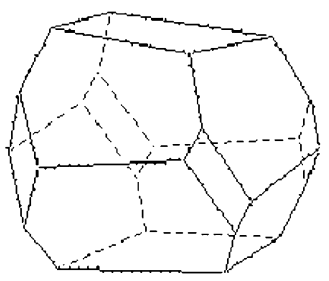

(c)

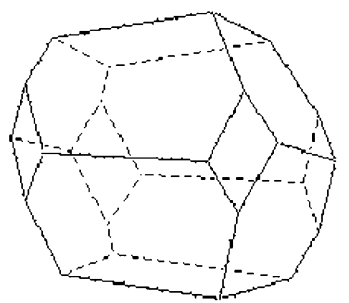

(b)

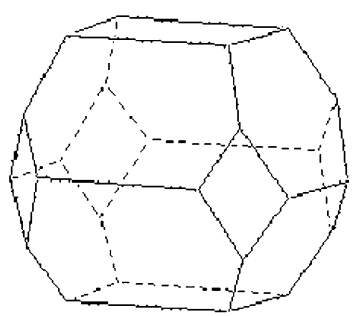

(d)
Figure 6

Lattice Voronoi-Dirichlet polyhedra. (a) The perfect body-centred cubic structure of tungsten at room-temperature. $(b)$ and $(c)$ The components of the asymmetric unit of cyclobutanol-I at $100 \mathrm{~K}$. (d) Cyclobutanol-II at $1.3 \mathrm{GPa}$. 
packing, as this is indicative of overall intermolecular contacts. The topological characteristics of packing in molecular crystal structures have been studied by Blatov and co-workers (Blatov et al., 2000). The coordination environment of a molecule in a crystal structure can be visualized using a Voronoi-Dirichlet polyhedron or VDP (Peresypkina \& Blatov, 2000a,b). The greater efficiency of packing in cyclobutanol-II (the high pressure phase) can be gauged by comparison of the lattice VDPs of the two phases. In both phases the molecular coordination number $(\mathrm{MCN})$ is 14 . Fourteen is the most commonly observed value in molecular structures and ideally the VDP is a cuboctahedron, as observed in the body-centred cubic structure of tungsten (Fig. $6 a)$; this VDP is characterized by a covering coefficient (Peresypkina \& Blatov, 2000a) of 1.46. The VDPs of the two independent molecules in cyclobutanol-I are shown in Figs. $6(b)$ and (c), and they clearly correspond to distorted versions of the cuboctahedron shown in Fig. 6(a). The covering coefficients are 1.98 and 2.00. The VDP of cyclobutanol-II (Fig. $6 d$ ) is still a distorted version of Fig. 6(a), but the distortion is less than for phase I, with a covering coefficient of 1.69. The change in the crystal structure of cyclobutanol on application of pressure can thus be considered to be driven by the adoption of a packing arrangement which more closely resembles that adopted in hard-sphere structures.

\section{Conclusions}

The structural changes exhibited between the low-temperature phase of cyclobutanol and its corresponding high-pressure phase are strongly paralleled by the changes we have observed previously between the low-temperature and highpressure phases of phenol and its halogenated derivatives 2chlorophenol and 4-fluorophenol (Oswald et al., 2005). The general structural change accompanying the helical to coplanar structural rearrangement of the molecules in these systems results in a marked improvement in packing efficiency and would appear to be driven by the steric hindrance of the phenyl groups. For cyclobutanol, an analogous affect appears to be influencing the high-pressure structural behaviour as the change of structure appears to be in response to the molecules adopting an arrangement analogous to the packing of hard spheres.

We would like to offer our thanks to S. A. Moggach for his help in the preparation of this paper. We also thank the EPSRC for funding this work and for supporting DRA through his EPSRC Advanced Fellowship.

\section{References}

Allan, D. R., Clark, S. J., Dawson, A., McGregor, P. A. \& Parsons, S. (2002). Acta Cryst. B58, 1018-1024.

Blatov, V. A., Shevchenko, A. P. \& Serezhkin, V. N. (2000). J. Appl. Cryst. 33, 1193.

Blessing, R. H. (1997). J. Appl. Cryst. 30, 421-426.

Boese, R. \& Nussbaumer, M. (1994). Correlations, Transformations and Interactions in Organic Chemistry, IUCr Crystallographic Symposia, Vol. 7, edited by D. W. Jones \& A. Katrusiak. Oxford University Press.

Brock, C. P. \& Duncan, L. L. (1994). Chem. Mater. 6, 1307-1312.

Dawson, A., Allan, D. R., Parsons, S. \& Ruf, M. (2004). J. Appl. Cryst. 37, 410-416.

Flack, H. D. (1983). Acta Cryst. A39, 876-881.

Merrill, L. \& Bassett, W. A. (1974). Rev. Sci. Instrum. 45, 290-294.

Oswald, I. D. H., Allan, D. R., Motherwell, W. D. S. \& Parsons, S. (2005). Acta Cryst. B61, 69-79.

Parsons, S. (2004). SHADE. The University of Edinburgh, Scotland.

Peresypkina, E. V. \& Blatov, V. A. (2000a). Acta Cryst. B56, 501-511.

Peresypkina, E. V. \& Blatov, V. A. (2000b). Acta Cryst. B56, 10351045.

Sheldrick, G. M. (2001). SADABS. University of Göttingen, Germany, and Bruker AXS, Madison, Wisconsin, USA.

Siemens (1993). SMART. Siemens Industrial Autom., Inc., Madison, Wisconsin, USA.

Siemens (1995). SAINT. Siemens Industrial Autom., Inc: Madison, Wisconsin, USA.

Sparks, R. A. (1999). Bruker AXS, Madison Wisconsin, USA.

Spek, A. L. (2001). PLATON. Utrecht University, The Netherlands. 